\title{
La importancia de las habilidades cognitivo-lingüísticas asociadas al estudio de la Astronomía
} desde la perspectiva del profesorado

\section{The importance of the cognitive-linguistic skills related to the study of the Astronomy from the teacher's view}

Susana García Barros, Cristina Martínez Losada

Departamento de Pedagoxía e Didáctica.

Universidade da Coruña

susg@udc.es, cmarl@udc.es

RESUMEN • El trabajo pretende conocer cómo valora el profesorado de Primaria y Secundaria en ejercicio y formación una serie de habilidades cognitivo-lingüísticas (descripción de hechos/fenómenos, de modelos, definición...). También se pretende conocer si los docentes establecen diferencias entre las habilidades expresadas genéricamente y en términos astronómicos, y si existen diferencias entre los grupos de docentes. Así mismos se analiza si los profesores identifican y valoran estas habilidades en actividades concretas. Los resultados muestran que los docentes valoran positivamente las habilidades, apreciándose diferencias entre las enunciadas genéricamente y en términos astronómicos y entre grupos de profesores. Los participantes tienen dificultades para identifican la habilidad cognitivo-lingüística exigidas en las actividades.

PALABRAS CLAVE: habilidades cognitivo-lingüísticas; Astronomía; ideas de los profesores; Primaria; Secundaria.

ABTRACT - The aim of this study is to find out how teachers of the last years of Primary and the first years of Secondary evaluate and what difficulties they find in relation to a series of cognitive-linguistic skills (description of facts,...). It also aims to find out whether teachers establish differences when the skills are expressed in a general sense or when they are associated to the subject of astronomy. Furthermore, it analyse if teachers identify and value these skills in specific activities. The results show that the teachers positively evaluate the skills presented, with changes being seen in terms of content. Differences between groups of teachers also were found. Participants have difficulties for identify the cognitive-linguistic skill that is demanded in the shown activities.

KEYWORDS: cognitive-linguistic skills; Astronomy; Primary; Secondary; teachers' ideas.

Fecha de recepción: enero 2012 • Aceptado: agosto 2012 


\section{INTRODUCCIÓN}

Recientemente se han introducido las competencias como los entes organizadores del currículo, definiéndose estas como la capacidad para poner en práctica de forma integrada en contextos y situaciones diversas, los conocimientos las habilidades y las actitudes personales adquiridas. Una de las competencias reconocidas es la científica que contempla, tanto las capacidades específicas asociadas, como los contenidos, las actitudes y el contexto personal/social (Cañas, Martín-Díaz y Nieda, 2007). Esta competencia no se consigue de forma aislada, reconociéndose específicamente la complementariedad de las competencias científica y lingüística (Pérez Esteve y Zayas, 2007; Prain, 2006). En esta línea, desde la enseñanza de las ciencias se viene defendiendo la necesidad de hablar, escribir y leer ciencias (Sanmartí, Izquierdo y García Rovira, 1999; Sanmartí, 2007), pues en este contexto, igual que ha ocurrido en la historia de la ciencia, el lenguaje y las ideas tienen un desarrollo paralelo (Sutton, 1996, 2003). Así, el lenguaje contribuye a hacer más científicos los modelos explicativos mentales del alumno y la maduración de los modelos promueve la evolución del lenguaje (Izquierdo y Aliberas, 2004).

Por otra parte, está ampliamente admitido que la adquisición del conocimiento científico se favorece con el intercambio de opiniones y la negociación de significados entre alumnos y profesor (Gómez, 2000). En este sentido el alumno deberá emplear textos diferentes conjugando tanto habilidades cognitivas, como lingüísticas (habilidades cognitivo-lingüísticas) (Jorba, 2000). Su promoción, al igual que ocurre con otros procedimientos, depende de su enseñanza a través de las actividades específicas (De Pro, 1998; Sanmartí, 2007) y por extensión de las decisiones del profesorado que dependen, en definitiva, de la importancia otorgada a este tipo de destrezas en el aprendizaje científico. Se ha venido destacando que el pensamiento y la acción docente, desarrollados a lo largo del tiempo, conforma una cultura profesional que dirige al profesor, constituyendo, en ocasiones, un obstáculo para la innovación (Pozo, Scheuer, Mateos y Pérez, 2006). Por ello, conocer el pensamiento docente puede servir de base para la reflexión, repercutiendo favorablemente en la formación del profesorado. Esta línea de investigación es amplia y variada, trabajos recientes se dirigen a conocer, cuales son las ideas profesionales de los futuros docentes (Benarroch y Marín, 2011) y como progresan (Fuentes, García Barros y Martínez Losada, 2009; Porlán et al., 2011). Otros realizados con docentes en ejercicio se dirigieron a conocer cómo valoran los procedimientos o actividades (C Martínez Losada y García Barros, 2005) (García Barros y Martínez losada, 2011), las actividades fuera del aula (museos) (Guisasola y Morentin, 2010), etc.

Con relación a las habilidades cognitivo-lingüísticas también se han realizado trabajos con profesores, dirigidos a identificar cuáles de estas habilidades se exigen en las actividades propuestas por los docentes universitarios de Psicopedagogía (Vázquez, Pelizza, Jakob y Rosales, 2007), encontrándose que la justificación y argumentación son menos consideradas que la descripción y la definición. Así mismo se dirigieron a establecer criterios para evaluar la presencia de habilidades cognitivo-lingüísticas en textos elaborados por profesores de química en formación, estableciendo su relación con el aprendizaje de conceptos (Ospina Quintero y Bonan, 2011). También se han analizado las cuestiones de evaluación planteadas por profesores de Primaria y Secundaria en formación, apreciándose que demandaban los distintos tipos de habilidades cognitivolinüísticas, aunque no siempre eran identificadas adecuadamente por sus autores/as (Martínez Losada, García Barros y Jiménez-Liso, 2011).

El trabajo que presentamos se orienta a profundizar en el pensamiento docente en cuanto al valor educativo otorgado a las habilidades cognitivo-lingüísticas, tanto en sentido general, como en relación a un tema específico -la Astronomía-. La elección de este tema responde básicamente a su presencia en el currículum (Solbes y Palomar, 2011) y a que constituye un tema idóneo para desarrollar diferentes habilidades cognitivo-lingüísticas, en la medida que atiende al ámbito fenomenológico descriptivo -qué se observa y qué cambia en el cielo- e interpretativo implicado en la justificación de hechos, mediante el uso de modelos. Concretamente se plantean las siguientes cuestiones: 
- ¿Cómo valoran los profesores de Primaria y Secundaria en ejercicio y en formación la importancia educativa de distintas habilidades cognitivo-lingüísticas enunciadas en términos generales y asociadas a la Astronomía? ¿Existen diferencias entre la valoración realizada por los distintos grupos de profesores?

- ¿La valoración que realizada el profesorado de las distintas habilidades está influenciada por el contenido?

- ¿Cómo valoran los docentes las habilidades cognitivo-lingüísticas concretas que demandan determinadas actividades dirigidas al estudio de la Astronomía?

\section{Habilidades cognitivo-lingüísticas}

Basándonos en las revisiones realizadas por Jorba (2000) dentro de las habilidades cognitivo-lingüísticas se contemplan los siguientes tipos: $a$ ) describir consiste en producir proposiciones o enunciados que enumeran cualidades, propiedades, etc., de objetos, hechos o fenómenos o incluso de modelos explicativos abstractos; $b$ ) definir supone expresar las características necesarias y suficientes para que un concepto no se pueda confundir con otro; $c$ ) justificar implica elaborar la expresión de una interpretación de fenómenos o acontecimientos vinculándolos con la teoría, y d) argumentar requiere construir los enunciados en base a pruebas con la intención de ser empleadas para convencer a otras personas.

La adquisición de habilidades cognitivo-lingüísticas no se realiza de manera independiente, sino que se relaciona con el desarrollo de habilidades cognitivas y el aprendizaje de contenidos curriculares. Así la adquisición de habilidades cognitivas, como por ejemplo analizar, comparar, clasificar, interpretar, deducir, etc., que están en la base del aprendizaje, posibilitan y concretan las habilidades cognitivolingüísticas que, a su vez, favorecen el aprendizaje de los contenidos curriculares. Así mismo, éste tiene un efecto potenciador de las habilidades cognitivo-lingüísticas y por extensión de las cognitivas (Jorba, 2000).

Si bien las habilidades cognitivo-lingüísticas son transversales a las distintas disciplinas, deben concretarse diferencialmente en cada una de ellas. En el caso de las ciencias de la naturaleza, que tiene como objetivo promover que el alumnado conozca los fenómenos del mundo que nos rodean desarrolle la capacidad de interpretarlos, desde modelos científicos cada vez más adecuados y complejos, y se desenvuelva responsablemente en la sociedad (Izquierdo \& Adúriz Bravo, 2003; Izquierdo, Sanmartí y Espinet, 1999), el estudiante tendrá que desarrollar las habilidades cognitivo-lingüísticas antes mencionadas, que le permitirán generar textos adecuados para cada finalidad. Así, el estudiante tendrá que describir los hechos/fenómenos y los modelos teóricos que exige la ciencia escolar, pues una buena descripción es la base necesaria para poder elaborar otros tipos de textos, como definiciones o justificaciones. Estas últimas requieren que se conecten hechos/fenómenos con el marco explicativo. Por ello, es importante insistir en este tipo de textos, sin duda más complejos que, a pesar de su importancia, tienen escasa presencia en las explicaciones del profesorados y en de los libros de texto, a pesar de que son frecuentemente exigidos en las evaluaciones (Izquierdo y Sanmartí, 2000).

\section{Astronomía}

La Astronomía es un tema que encierra gran interés educativo y despierta curiosidad entre el alumnado (Trumper, 2006). En este sentido, la European Association form Astronomy in Europe'schools declara que la Astronomía debería introducirse ya en los primeros niveles educativos, haciendo especial énfasis en las observaciones de los objetos que se ven en el cielo y la enseńanza de los modelos que los explican. El aprendizaje de la Astronomía, debe por tanto, al igual que ocurre en otros temas de ciencias, promover el conocimiento profundo de los fenómenos observables (Navarro, 2011; Shen y Confrey, 
2010) y su explicación teórica, resultando especialmente importante insistir en la experiencia empírica, especialmente en los niveles más elementales (Harlen y Qualter, 2009). Sin embargo, la enseñanza de la Astronomía y los textos escolares que se emplean, muestran deficiencias (Vosniadou, d1991; García Barros, Martínez Losada, Mondelo y Vega, 1997; Martínez Peña y Gil, 2001). Incluso en los manuales más recientes de Primaria se detecta una escasa atención al contenido observacional y a su vinculación entre éste y el modelo (Navarro, 2009). Lo indicado está influido por las propias concepciones docentes, que se sienten incómodos enseñando el movimiento aparente del sol, pues consideran que inducen al niño a realizar una interpretación geocéntrica errónea (Shen y Confrey, 2010).

La enseñanza de la Astronomía requiere, al igual que otros temas, seleccionar adecuadamente los fenómenos sobre los que hacer las preguntas teóricas y organizar así los contenidos a enseñar de manera que vayan de lo concreto a lo abstracto, de lo simple a lo complejo y de lo próximo a lo lejano (Sanmartí, 2002). En este sentido, la utilización del paso del tiempo puede servir de hilo conductor para organizar los contenidos astronómicos (paso del día, del mes, del año) (Martínez Losada y García Barros, 2010). En cada uno de estos tres apartados se debe promover el uso de habilidades cognitivolingüísticas. Así, la descripción de fenómenos y modelos, junto a la justificación, en cuanto vinculan observación y modelo mediante la interpretación, resultan esenciales para conocer qué pasa en el cielo en cada espacio de tiempo y por qué pasa.

\section{METODOLOGÍA}

En este estudio han participado 155 profesores: 59 profesionales con experiencia en $2 .^{\circ}$ y $3 .^{\text {er }}$ ciclo de Primaria; 54 profesores del primer ciclo de ESO; 21 profesores de Primaria en formación -alumnos de magisterio- y 21 futuros docentes de Secundaria -alumnos del máster de Secundaria.

La recogida de datos se realizó mediante una encuesta. Los profesores en ejercicio, que accedieron voluntariamente a participar en el estudio, pertenecían a distintos centros de la provincia de A Coruña. En ningún caso el número de respuestas por centro fue superior a dos profesores. Los docentes en formación también contestaron voluntariamente a la encuesta. Estos últimos habían trabajado en las asignaturas de Didáctica de las Ciencias la importancia educativa de las habilidades cognitivolingüística s. Así mismo, habían analizado científica y didácticamente los fenómenos astronómicos y su explicación teórica.

La encuesta constaba de dos partes. En la primera los participantes debían valorar de 1 a 5 la importancia educativa de una serie de habilidades cognitivo-lingüísticas: descripción de fenómenos o hechos (DF), descripción de modelos teóricos (DM), definición (Def) y justificación de hechos/fenómenos (J). Estas habilidades se redactaron tanto con enunciados genéricos acompañados de ejemplos, como con enunciados astronómicos (anexo). En este segundo caso los enunciados implican cada una de las habilidades citadas, asociadas a aspectos astronómicos de distinto nivel de dificultad: a) relativos a los cambios diarios (descripción del cambio aparente del Sol, descripción y definición de la rotación terrestre, justificación del cambio aparente del Sol empleando el modelo de rotación) y b) relativos a los cambios anuales (descripción de cambios astronómicos anuales en nuestras latitudes, descripción y definición de la traslación terrestre y justificación de los cambios anuales empleando el modelo). En la segunda parte de la encuesta, de carácter abierto, se presentaban tres actividades, centradas en el estudio de la Luna, que exigían habilidades diferentes: 1) definición de satélite; 2) descripción de un fenómeno -cambios de posición aparentes en 24 horas-, y 3) justificación de los cambios, empleando el modelo Sol/Tierra/Luna. Los participantes debían valorar justificadamente el interés educativo de las habilidades cognitivo-lingüísticas exigidos en cada actividad, que denominamos habilidades relacionadas con la comunicación, para evitar tecnicismos y facilitar la comprensión. La encuesta en 
su conjunto, antes de ser empleada, fue revisada y discutida con 4 profesores expertos de Educación Primaria y Secundaria.

Con el fin de detectar diferencias en las valoraciones otorgadas a los distintos enunciados entre los distintos colectivos se empleó el $\chi^{2}$ de Pearson, considerándose únicamente las valoraciones máximas (4 o 5). Concretamente se comparan los grupos de profesores: $a$ ) de Primaria y de Secundaria en ejercicio; b) de Primaria y de Secundaria en formación; $c$ ) de Primaria en ejercicio y formación, y d) de Secundaria en ejercicio y formación. Por otra parte, para identificar diferencias dentro de cada grupo de profesores, se empleó el test de McNemar, considerando también las puntuaciones máximas. Concretamente, en cada grupo se analizó si los sujetos establecen diferencias entre las cuatro habilidades cognitivo-lingüística s expresadas: $a$ ) en términos generales; $b$ ) en términos relacionados con cambios astronómicos diarios, $\mathrm{y} c$ ) en términos relacionados con cambios astronómicos anuales. Además se analizaron las diferencias entre el valor otorgado a cada habilidad cognitivo-lingüística enunciada genéricamente y a su homóloga expresada en términos astronómicos (cambios diarios y anuales respectivamente). Para los análisis estadísticos se utilizó el programa SPSS (valor de significatividad p<0,05). Así mismo, se hizo un estudio individualizado de las valoraciones realizadas por cada participante. Aquí se consideraron todas las puntuaciones, no solo las de mayor valor, estableciéndose tipos de respuesta en función de la menor, mayor o igual importancia que los sujetos otorgan a unos enunciados en comparación con otros. Los tipos de respuestas se establecieron para las habilidades cognitivo-lingüísticas asociadas a los enunciados genéricos y a cada uno de los astronómicos (cambios diarios/anuales) (ver tipos de respuesta en tabla 3).

Las valoraciones justificadas, positivas y negativas, de las habilidades demandadas por las actividades se clasificaron en función de que la valoración haga referencia: a) explícitamente a la habilidad cognitivo-lingüística exigida en la actividad; $b$ ) a aspectos conceptuales tratados en la actividad u a otras habilidades, $\mathrm{y} c$ ) a otros comentarios. El análisis fue realizado independientemente por dos investigadoras, discutiendo las posibles discrepancias.

\section{RESULTADOS}

La mayoría de los enunciados incluidos en la encuesta reciben puntuación máxima (4 ó 5) por más del 50\% de los docentes. Las habilidades menos consideradas son la definición en términos genéricos (G-Def), en todos los grupos menos en el de ESO en ejercicio, donde la descripción del fenómeno asociada al cambio diario (D-DF) es la habilidad menos considerada (tabla 1). El análisis estadístico $\left(\chi^{2}\right.$ de Pearson) permitió identificar las siguientes diferencias entre grupos:

- entre los profesores en ejercicio, los de ESO valoran más que los de Primaria la definición en términos genéricos $(\mathrm{G}-\mathrm{Def})(\mathrm{p}=0,019)$ y la descripción de modelos, tanto en términos genéri$\cos (\mathrm{G}-\mathrm{DM})(\mathrm{p}=0,005)$ como asociada a cambios anuales (A-DM) $(\mathrm{p}=0,09)$, mientras que los de Primaria consideran más la descripción del modelo asociado a los cambios diarios (D-DM) $(\mathrm{p}=0,042)$;

- entre profesores en formación de ESO y Primaria no se hallaron diferencias;

- entre los profesores en ejercicio y en formación, estos últimos realizan valoraciones más altas, concretamente los futuros maestros consideran más la descripción del modelo (G-DM) $(\mathrm{p}=0,047)$ y la justificación en términos generales $(\mathrm{G}-\mathrm{J})(\mathrm{p}=0,04)$ y con relación a cambios anuales (A-J) ( $\mathrm{p}=0,004)$, mientras que los profesionales solo dan más importancia a la descripción de estos últimos (A-DF) ( $\mathrm{p}=0,006)$; por otra parte, docentes de Secundaria en formación valoran más que los profesionales la justificación en términos genéricos (G-J) ( $\mathrm{p}=0,019)$. 
Tabla 1.

Profesores que otorgan máxima valoración (4 o 5) a los distintos enunciados

\begin{tabular}{|c|c|c|c|c|c|}
\hline \multirow{2}{*}{\multicolumn{2}{|c|}{$\begin{array}{c}\text { ENUNCIADOS } \\
\text { Primaria } \\
\mathrm{N}=59\end{array}$}} & \multicolumn{2}{|c|}{ Prof. en ejercicio } & \multicolumn{2}{|c|}{ Prof. en formación } \\
\hline & & \multirow{2}{*}{$\begin{array}{c}\text { ESO } \\
\mathrm{N}=54 \\
49 \\
83,1 \% \\
\end{array}$} & \multirow{2}{*}{$\begin{array}{c}\text { Primaria } \mathrm{n}=21 \\
45 \\
83,3 \%\end{array}$} & \multirow{2}{*}{$\begin{array}{c}\text { ESO } \\
\mathrm{N}=21\end{array}$} & \multirow[b]{2}{*}{$\begin{array}{c}17 \\
81,0 \%\end{array}$} \\
\hline \multirow{4}{*}{ 王 } & G-DF & & & & \\
\hline & G-DM & $\begin{array}{l}34^{(1)(2)} \\
57,6 \%\end{array}$ & $\begin{array}{c}44^{(1)} \\
81,5 \%\end{array}$ & $\begin{array}{l}17^{(2)} \\
81,0\end{array}$ & $\begin{array}{c}19 \\
90,5 \%\end{array}$ \\
\hline & G-Def. & $\begin{array}{c}28^{(1)} \\
47,5 \% \\
\end{array}$ & $\begin{array}{c}37^{(1)} \\
68,5 \% \\
\end{array}$ & $\begin{array}{c}6 \\
28,6 \%\end{array}$ & $\begin{array}{c}10 \\
47,6 \% \\
\end{array}$ \\
\hline & G-J & $\begin{array}{c}38^{(2)} \\
64,4 \% \\
\end{array}$ & $\begin{array}{c}41^{(3)} \\
75,9 \% \\
\end{array}$ & $\begin{array}{c}20^{(2)} \\
95,2 \%\end{array}$ & $\begin{array}{c}20^{(3)} \\
95,2 \%\end{array}$ \\
\hline \multirow{4}{*}{ 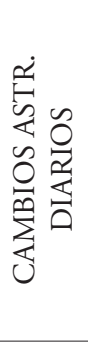 } & D-DF & $\begin{array}{c}43 \\
72,9 \% \\
\end{array}$ & $\begin{array}{c}32 \\
59,3 \% \\
\end{array}$ & $\begin{array}{c}16 \\
76,2 \% \\
\end{array}$ & $\begin{array}{c}16 \\
76,2 \% \\
\end{array}$ \\
\hline & D-DM & $\begin{array}{c}53^{(1)} \\
89,8 \% \\
\end{array}$ & $\begin{array}{c}41^{(1)} \\
75,9 \%\end{array}$ & $\begin{array}{c}21 \\
100,0 \% \\
\end{array}$ & $\begin{array}{c}19 \\
90,5 \%\end{array}$ \\
\hline & D-Def. & $\begin{array}{c}49 \\
83,1 \% \\
\end{array}$ & $\begin{array}{c}39 \\
72,2 \% \\
\end{array}$ & $\begin{array}{c}18 \\
85,7 \% \\
\end{array}$ & $\begin{array}{c}15 \\
71,4 \% \\
\end{array}$ \\
\hline & D-J & $\begin{array}{c}44 \\
74,6 \% \\
\end{array}$ & $\begin{array}{c}36 \\
66,7 \% \\
\end{array}$ & $\begin{array}{c}18 \\
85,7 \% \\
\end{array}$ & $\begin{array}{c}17 \\
81,0 \% \\
\end{array}$ \\
\hline \multirow{4}{*}{ 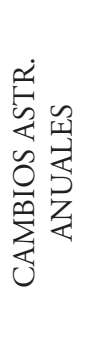 } & A-DF & $\begin{array}{l}39^{(2)} \\
66,1\end{array}$ & $\begin{array}{c}40 \\
74,1 \% \\
\end{array}$ & $\begin{array}{c}7^{(2)} \\
33,3 \% \\
\end{array}$ & $\begin{array}{c}12 \\
57,1 \%\end{array}$ \\
\hline & A-DM & $\begin{array}{c}30^{(1)} \\
50,8 \%\end{array}$ & $\begin{array}{c}40^{(1)} \\
74,1 \% \\
\end{array}$ & $\begin{array}{c}15 \\
71,4 \% \\
\end{array}$ & $\begin{array}{c}15 \\
71,4 \% \\
\end{array}$ \\
\hline & A-Def. & $\begin{array}{c}49 \\
83,1 \% \\
\end{array}$ & $\begin{array}{c}40 \\
74,1 \% \\
\end{array}$ & $\begin{array}{c}15 \\
71,4 \%\end{array}$ & $\begin{array}{c}18 \\
85,7 \% \\
\end{array}$ \\
\hline & A-J & $\begin{array}{c}32^{(2)} \\
54,2 \%\end{array}$ & $\begin{array}{c}36 \\
66,7 \% \\
\end{array}$ & $\begin{array}{c}19^{(2)} \\
90,5 \%\end{array}$ & $\begin{array}{c}17 \\
81,0 \%\end{array}$ \\
\hline
\end{tabular}

Diferencias significativas entre: (1) los profesores de Primaria y Secundaria en ejercicio; (2) los profesores de Primaria en ejercicio y en formación, y (3) los profesores de ESO en ejercicio y en formación (prueba Chi cuadrado, p<0,05).

El análisis realizado en cada uno de los grupos de profesores muestra que existen diferencias significativas en la valoración máxima otorgada a las habilidades (tabla 2). Los profesionales de Primaria son los que establecen más diferencias, así al comparar la valoración de las distintas habilidades se aprecia que: $a$ ) entre las enunciadas genéricamente, la descripción de fenómenos (G-DF) se considera más que otras (G-DM y G-Def); b) entre las enunciadas en relación a cambios diarios, la descripción del modelo, (D-DM) se valora más que la del fenómeno y la justificación, y $c$ ) entre las relacionadas con cambios anuales, la definición (A-Def) es la más valorada, y la descripción del fenómeno (A-DF) más que la del modelo. Al comparar la valoración de cada habilidad en términos genéricos y astronómicos se observa que se consideran más estos últimos. En concreto, la descripción del modelo, asociada a cambios diarios (D-DM), y las definiciones, asociadas a cambios diarios (D-Def) y anuales (A-Def), se valoran más que sus homólogas genéricas.

Los docentes de ESO en ejercicio solo establecen diferencias entre las habilidades cognitivo-lingüística s enunciadas en términos astronómicos. Priorizan la descripción del modelo (D-DM), asociada a cambios diarios, frente a la de fenómenos, mientras la definición (A-Def) es la habilidad menos considerada de las asociadas a cambios anuales. Por otra parte, se aprecia que la definición se considera más 
en términos astronómicos (D-Def; ADef) que en términos genéricos y la descripción de fenómenos más en términos genéricos $(\mathrm{G}-\mathrm{DF})$ que en relación a cambios diarios.

Los futuros maestros diferencian entre las habilidades enunciadas en términos genéricos, la definición (G-Def) es la menos considerada, y también entre las asociadas a los cambios anuales -aquí la descripción de fenómenos (A-DF) es la menos valorada-. Como en otros grupos, la definición se considera más en términos astronómicos (D-Def; A-Def) que genéricos, y la descripción de fenómenos más en términos genéricos (G-DF) que asociada a cambios anuales. Los profesores de ESO en formación solo establecen una diferencia, la definición con relación a cambios anuales (A-Def) se valora más que en términos genéricos.

\section{Tabla 2}

Diferencias significativas halladas al comparar la valoración:

a) de las 4 habilidades cognitivo-lingüísticas expresadas en términos genéricos y en términos astronómicos, y b) de cada habilidad en términos genéricos con su homóloga en términos astronómicos

(Test de Mc Nemar, $\mathrm{p}<0,05$ )

\begin{tabular}{|c|c|c|c|c|}
\hline \multirow{2}{*}{\multicolumn{2}{|c|}{$\begin{array}{c}\text { COMPARACIONES } \\
\text { ENTRE } \\
\text { PRIMARIA }\end{array}$}} & \multicolumn{2}{|c|}{ PROFESORES EJERCICIO } & \multirow[t]{2}{*}{ PROF. FORMACIÓN } \\
\hline & & ESO & PRIMARIA & \\
\hline \multicolumn{2}{|c|}{ Enunciados genéricos } & $\begin{array}{l}\text { G-DF>G-DM; } p=0,001 \\
\text { G-DF>G-Dfe; } p<0,000\end{array}$ & & $\begin{array}{l}\text { G-DF>G-Def; } p=0,002 \\
\text { G-DM }>\text { G-Def; } p=0,007 \\
\text { G-J > G-Def; } p=0,000\end{array}$ \\
\hline \multirow{2}{*}{ 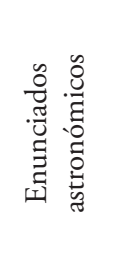 } & $\begin{array}{l}\text { Cambios } \\
\text { diarios }\end{array}$ & $\begin{array}{l}\mathrm{D}-\mathrm{DM}>\mathrm{D}-\mathrm{DF} ; \mathrm{p}=0,031 \\
\mathrm{D}-\mathrm{DM}>\mathrm{D}-\mathrm{J} ; \mathrm{p}=0,022\end{array}$ & $\mathrm{D}-\mathrm{DM}>\mathrm{D}-\mathrm{DF} ; \mathrm{p}=0,049$ & \\
\hline & $\begin{array}{l}\text { Cambios } \\
\text { anuales }\end{array}$ & $\begin{array}{l}\text { A-DF }>A-D M ; p=0,031 \\
\text { A-Def }>\text { A-DF; } p=0,035 \\
\text { A-Def }>\text { A-DM; } p<0,000 \\
\text { A-Def }>\text { A-J; } p<0,000\end{array}$ & $\begin{array}{l}\text { A-DF }>\text { A-Def; } p<0,000 \\
\text { A-DM }>\text { A-Def; } p<0,000 \\
\text { A-J }>\text { A-Def; } p<0,000\end{array}$ & $\begin{array}{l}\text { A-DM>A-DF; } p=0,008 \\
\text { A-Def }>A-D F ; p=0,001 \\
\text { A-J }>A-D F ; p=0,004\end{array}$ \\
\hline \multirow{2}{*}{ 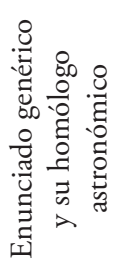 } & $\begin{array}{l}\text { Cambios } \\
\text { diarios }\end{array}$ & $\begin{array}{l}\text { D-DM }>\text { G-DM; } p<0,000 \\
\text { D-Def }>\text { G-Dfe; } p<0,000\end{array}$ & $\begin{array}{l}\text { G-DF }>D-D F ; p=0,004 \\
D-D e f>G-D f e ; P<0,000\end{array}$ & D-Def $>$ G-Df; $p<0,000$ \\
\hline & $\begin{array}{l}\text { Cambios } \\
\text { anuales }\end{array}$ & A-Def $>$ G-Dfe; $p<0,000$ & A-Def $>$ G-Dfe; $p<0,000$ & $\begin{array}{l}\text { G-DF }>A-D F ; p=0,001 \\
\text { A-Def }>\text { G-Dfe; } p<0,000\end{array}$ \\
\hline
\end{tabular}

Nota: En el grupo de profesores de ESO en formación se detecta una única diferencia: A-Def> G-Def; p=0,012.

El análisis de las puntuaciones dadas, por cada participante, a las cuatro habilidades cognitivolingüísticas, asociadas a los tres enunciados (generales, cambios diarios y anuales), permite identificar distintos tipos de respuestas en función de que: $a$ ) todas las habilidades tengan igual valor; $b$ ) tres igual valor, superior a una cuarta; $c$ ) dos igual valor, superior a las otras, y d) una el mayor valor. Dada la variedad de tipos, destacamos solo los más frecuentes, 13 en total, expresados por más del 10\% de los componentes de algún grupo (tabla 3). Concretamente, respecto a los enunciados genéricos, se identificaron siete tipos. El caracterizado por otorgar igual valoración a tres habilidades, superior a la definición $(\mathrm{DF}=\mathrm{DM}=\mathrm{J}>\mathrm{Def}$ ), se detecta en todos los grupos, menos en el de profesores de ESO en ejercicio, mientras que los otros tipos fueron identificados solo en dos o en un único colectivo. Con relación a los cambios diarios, se identificaron cuatro tipos de respuestas. El caracterizado por la valoración uniforme es frecuente en todos los grupos. Los tipos caracterizados por la menor valoración de 
la descripción de fenómenos $(\mathrm{DM}=\mathrm{Def}=\mathrm{J}>\mathrm{DF})$ y por la priorización de la descripción del modelo (DM> DF; Def y J) son frecuentes en tres colectivos. Con relación a los cambios anuales se identificaron seis tipos de respuestas y ninguno de ellos resultó frecuente en más de dos grupos de profesores.

Conviene indicar que, exceptuando las valoraciones homogéneas, los tipos de respuestas frecuentes fueron diferentes según el tipo de enunciados al que se referían las habilidades cognitivo-lingüísticas, detectándose solo dos coincidencias en este sentido: a) el tipo que prioriza la justificación frente al resto ( $>$ >DF;DM;Def), identificado en términos genéricos y en relación a cambios anuales y $b$ ) el que otorga igual valor a todas las habilidades excepto a la descripción de fenómenos (DM=Def=J>DF), identificado en cambios diarios y anuales. Además, excepto uno, todos los tipos de respuestas frecuentes asociadas a los enunciados genéricos se caracterizan por priorizar la descripción de fenómenos frente a otra/s habilidad/es. Sin embargo, en los relacionados con enunciados astronómicos ocurre lo contrario -excepto uno, todos priorizan otras habilidades frente a la citada descripción.

Tabla 3

Tipos de respuestas más frecuentes con relación a la valoración

de las habilidades cognitivo-lingüísticas enunciadas en términos genéricos y astronómicos.

\begin{tabular}{|c|c|c|c|c|c|c|c|c|c|c|c|c|c|}
\hline \multirow{3}{*}{$\begin{array}{c}\text { TIPOS DE } \\
\text { RESPUESTA }\end{array}$} & & \multicolumn{4}{|c|}{ ENUNCIADO GENÉRICO } & \multicolumn{4}{|c|}{ CAMBIO DIARIO } & \multicolumn{4}{|c|}{ CAMBIO ANUAL } \\
\hline & \multicolumn{2}{|c|}{ Prof. ejercicio } & \multicolumn{2}{|c|}{ Prof. formación } & \multicolumn{2}{|c|}{ Prof. ejercicio } & \multicolumn{2}{|c|}{ Prof. formación } & \multicolumn{2}{|c|}{ Prof. ejercicio } & \multicolumn{2}{|c|}{ Prof. formación } & \\
\hline & $\begin{array}{c}\text { Pri. } \\
\mathrm{n}=59\end{array}$ & \begin{tabular}{|l|}
$\mathrm{ESO}$ \\
$\mathrm{n}=54$ \\
\end{tabular} & $\begin{array}{c}\text { Pri. } \\
\mathrm{n}=21\end{array}$ & $\begin{array}{l}\text { ESO } \\
\mathrm{n}=21\end{array}$ & $\begin{array}{c}\text { Pri. } \\
\mathrm{n}=59\end{array}$ & $\begin{array}{l}\mathrm{ESO} \\
\mathrm{n}=54\end{array}$ & $\begin{array}{c}\text { Pri. } \\
\mathrm{n}=21\end{array}$ & $\begin{array}{l}\text { ESO } \\
\mathrm{n}=21\end{array}$ & $\begin{array}{c}\text { Pri. } \\
\mathrm{n}=59\end{array}$ & $\begin{array}{l}\text { ESO } \\
\mathrm{n}=54\end{array}$ & $\begin{array}{c}\text { Pri. } \\
\mathrm{n}=21\end{array}$ & $\begin{array}{l}\text { ESO } \\
n=21\end{array}$ & \\
\hline A & $\mathrm{DF}=\mathrm{DM}=\mathrm{Def}=\mathrm{J}$ & & $\begin{array}{c}9 \\
16,7 \% \\
\end{array}$ & & $\begin{array}{c}3 \\
14,3 \% \\
\end{array}$ & $\begin{array}{c}19 \\
32,2 \% \\
\end{array}$ & $\begin{array}{c}18 \\
33,3 \% \\
\end{array}$ & $\begin{array}{c}6 \\
28,6 \% \\
\end{array}$ & $\begin{array}{c}4 \\
19,0 \% \\
\end{array}$ & $\begin{array}{c}10 \\
16,9 \% \\
\end{array}$ & $\begin{array}{c}17 \\
31,5 \% \\
\end{array}$ & & \\
\hline \multirow{3}{*}{ B } & $\mathrm{DF}=\mathrm{DM}=\mathrm{Def}>\mathrm{J}$ & & $\begin{array}{c}8 \\
14,8 \% \\
\end{array}$ & & & & & & & & & & \\
\hline & $\mathrm{DF}=\mathrm{DM}=\mathrm{J}>\mathrm{Def}$ & $\begin{array}{c}8 \\
13,6 \%\end{array}$ & & $\begin{array}{c}5 \\
23,8 \% \\
\end{array}$ & $\begin{array}{c}6 \\
28,6 \% \\
\end{array}$ & & & & & & & & \\
\hline & $\mathrm{DM}=\mathrm{Def}=\mathrm{J}>\mathrm{DF}$ & & & & & \begin{tabular}{|c|}
11 \\
$18,6 \%$ \\
\end{tabular} & $\begin{array}{c}10 \\
18,5 \% \\
\end{array}$ & & $\begin{array}{c}3 \\
14,3 \\
\end{array}$ & & & & $\begin{array}{c}4 \\
19,0 \% \\
\end{array}$ \\
\hline \multirow{5}{*}{$\mathrm{C}$} & $\mathrm{DF}=\mathrm{Def}>\mathrm{DM} ; \mathrm{J}$ & & & & & & & & & $\begin{array}{c}6 \\
10,2 \% \\
\end{array}$ & & & \\
\hline & $\mathrm{DF}=\mathrm{DM}>\mathrm{Def} ; \mathrm{J}$ & $\begin{array}{c}7 \\
11,9 \%\end{array}$ & & & & & & & & & & & \\
\hline & $\mathrm{DF}=\mathrm{J}>\mathrm{Def} ; \mathrm{DM}$ & & & $\begin{array}{c}6 \\
28,6 \% \\
\end{array}$ & $\begin{array}{c}3 \\
14,3 \%\end{array}$ & & & & & & & & \\
\hline & $\mathrm{DM}=\mathrm{Def}>\mathrm{DF} ; \mathrm{J}$ & & & & & & $\begin{array}{c}7 \\
13,0 \% \\
\end{array}$ & $\begin{array}{c}3 \\
14,3 \% \\
\end{array}$ & & & & & \\
\hline & Def $=\mathrm{J}>\mathrm{DF} ; \mathrm{DM}$ & & & & & & & & & & & $\begin{array}{c}6 \\
28,6 \%\end{array}$ & \\
\hline \multirow{4}{*}{$\mathrm{D}$} & DF $>$ DM;Def ;J & \begin{tabular}{|c|}
16 \\
$27,1 \%$ \\
\end{tabular} & & & $\begin{array}{c}3 \\
14,3 \%\end{array}$ & & & & & & & & \\
\hline & DM>DF; Def; J & & & & & & $\begin{array}{c}6 \\
11,1 \% \\
\end{array}$ & \begin{tabular}{|c|}
4 \\
$19,0 \%$ \\
\end{tabular} & $\begin{array}{c}3 \\
14,3 \% \\
\end{array}$ & & & & \\
\hline & Def>DF;DM;J & & & & & & & & & $\begin{array}{c}12 \\
20,3 \%\end{array}$ & & & $\begin{array}{c}3 \\
14,3 \%\end{array}$ \\
\hline & J>DF;DM;Def & & & $\begin{array}{c}3 \\
14,3 \% \\
\end{array}$ & & & & & & & & \begin{tabular}{|c|}
6 \\
$28,6 \%$ \\
\end{tabular} & $\begin{array}{c}4 \\
19,0 \%\end{array}$ \\
\hline
\end{tabular}

A.Todas las habilidades tienen igual valor; B. Tres igual valor, superior a una cuarta; C. Dos igual valor, superior a las otras, y D. Una el mayor valor.

Presentamos ahora la valoración de las habilidades cognitivo-lingüísticas exigidas en las actividades de la encuesta. Todos los docentes en formación realizaron una valoración justificada, aunque solo un reducido número de profesionales lo hizo, entre 15 y 19 en cada actividad. La mayoría de los docentes 
que justificaron su opinión dan una valoración positiva, siendo la primera y la tercera actividad ligeramente menos consideradas (tabla 4).

Tabla 4

Profesores que valoran positivamente y de forma justificada las habilidades cognitivo-lingüísticas que demandan las actividades sobre el estudio de la Luna

\begin{tabular}{|c|c|c|c|c|}
\hline \multirow[b]{2}{*}{ ACTIVIDADES } & \multicolumn{2}{|c|}{ Profesores en ejercicio } & \multicolumn{2}{|c|}{ Profesores en formación } \\
\hline & $\begin{array}{c}\text { Primaria } \\
\mathrm{n}_{1}=17 \\
\mathrm{n}_{2}=15 \\
\mathrm{n}_{3}=17\end{array}$ & $\begin{array}{c}\mathrm{ESO} \\
\mathrm{n}_{1}=18 \\
\mathrm{n}_{2}=19 \\
\mathrm{n}_{3}=18\end{array}$ & $\begin{array}{c}\text { Primaria } \\
\mathrm{n}=21\end{array}$ & $\begin{array}{l}\text { ESO } \\
n=21\end{array}$ \\
\hline 1. ${ }^{a}$ Definición & $14(82,35 \%)$ & $18(100 \%)$ & $16(76,19 \%)$ & $17(80,95 \%)$ \\
\hline 2. ${ }^{a}$ Descripción de un fenómeno & $14(93,33 \%)$ & $18(94,12 \%)$ & $21(100 \%)$ & $20(95,24 \%)$ \\
\hline 3. Justificación & $12(70,59 \%)$ & $17(94,44 \%)$ & $19(90,48 \%)$ & $21(100 \%)$ \\
\hline
\end{tabular}

$\mathrm{n}_{1}, \mathrm{n}_{2,} \mathrm{n}_{3}$. Corresponden al número de sujetos de cada colectivo que valoran de forma justificada las actividades $1 . .^{\mathrm{a}}, 2 .^{\mathrm{a}}$ y $3 .^{\mathrm{a}}$ respectivamente.

En la primera actividad (figura 1), alrededor de la mitad de los sujetos de los distintos grupos que hace una valoración positiva y justificada se refieren a la definición (ejemplos de respuestas textuales identificando al profesor):

- Clasificar las cosas y ser capaces de definirlas es fundamental (ESO-ejer.55)

- Es importante que los niños vayan aprendiendo a definir conceptos que les ayuden a ir entendiendo poco a poco el mundo (Pri-ejer.108)

El resto se refiere a aspectos conceptuales implicados en la actividad:

- Es muy interesante para la enseñanza de los distintos tipos de astros que existen (Pri-ejer.107)

- Gran interés educativo para comprender el sistema solar y los elementos que lo integran (ESO-ejer.40)

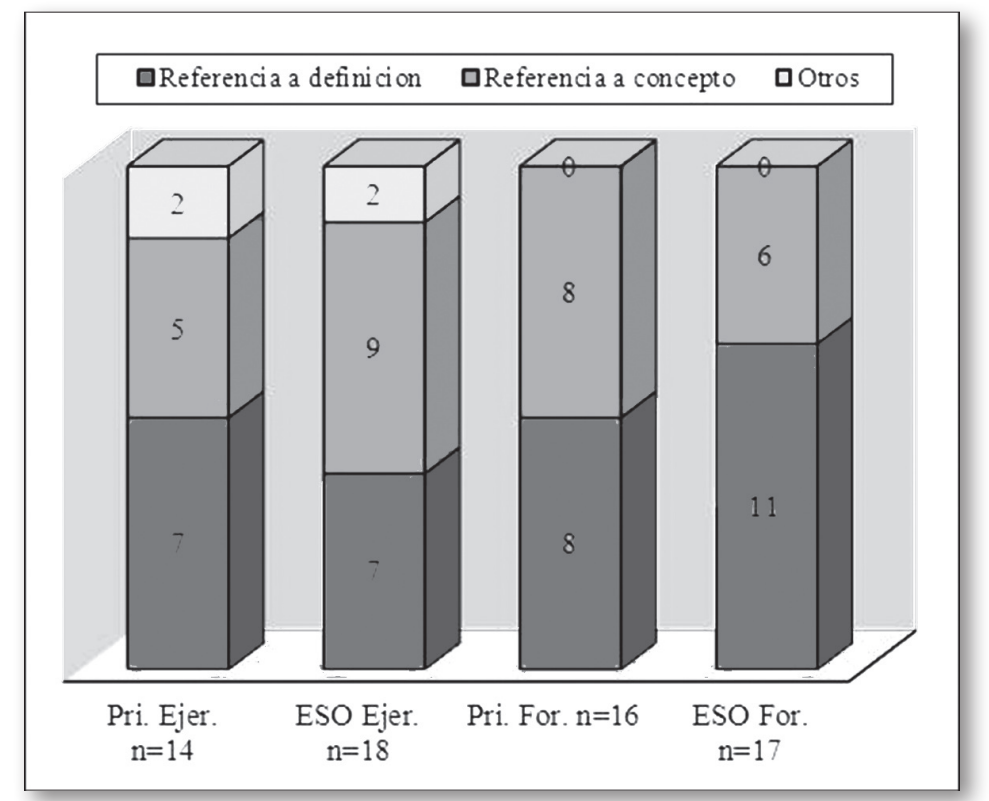

Fig. 1. Justificaciones de los profesores al valorar positivamente la actividad 1 . 
Más de la mitad de los 12 sujetos que emiten una valoración negativa señala la definición, matizando incluso la contraposición entre definir y entender.

- El interés de definir conceptos sobre astronomía no lo considero de alta importancia (ESO-for.122)

- Bajo porque promueve solo una definición, lo que no significa que lo entiendan (Pri-for.145)

El resto de las respuestas corresponde a la categoría de otros, refiriéndose incluso al carácter negativo de la memorización:

- Esta pregunta solo fomenta el estudio memorístico, no el aprendizaje significativo (ESO-for.125).

En la actividad dos son muy escasos los participantes (ninguno en el grupo de maestros en ejercicio y menos de la tercera parte en los otros), que emitiendo valoración positiva, se refieren a la habilidad exigida (descripción del fenómeno) (figura 2).

- Esta actividad exige al alumno realizar una descripción, pero además recoge un aspecto clave del método cientifico que es la observación (ESO-for.119)

La mayoría señala otras habilidades (la observación) o aportan justificaciones genéricas (otros):

- El interés educativo de la observación es fundamental (Pri-ejer.60).

- Actividad que fomenta que el alumno reflexione, piense y razone (ejemplo de otros) (ESO-for.115).

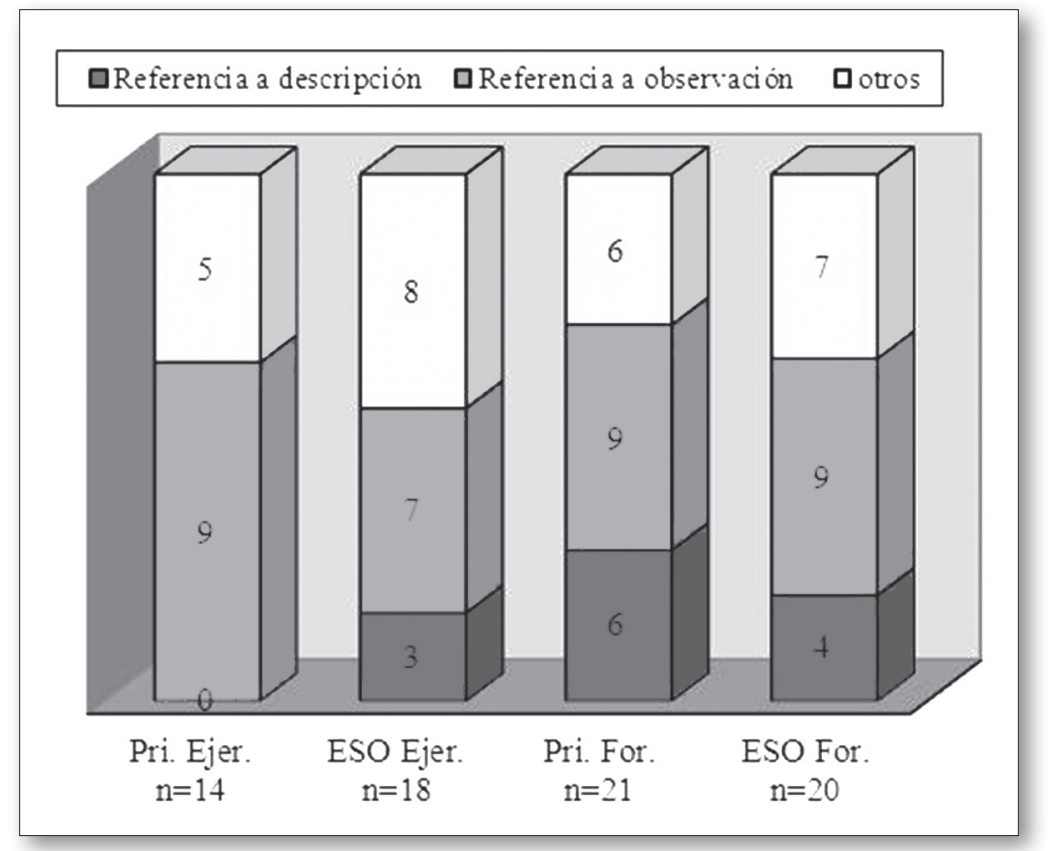

Fig. 2. Justificaciones de los profesores al valorar positivamente la actividad 2

Solo uno de los tres participantes que valoran negativamente la habilidad de esta actividad, menciona la descripción:

- Son solo descripciones (ESO-for.129).

El resto de las respuestas se catalogan como otros, y se asocian a problemas técnicos/metodológicos: 
- Interés escaso, por lo que conlleva trabajar con 25 alumnos en el aula. Cuesta mantener su atención (ESO-ejer.40).

En la actividad tres, también son escasos los participantes que, realizando una valoración positiva, se refieren a la habilidad exigida (justificación/explicación). En el grupo de profesores de Secundaria en formación se detectan más este tipo de respuestas, aunque apenas superan el $50 \%$.

- Con este modelo los alumnos pueden explicar fácilmente el movimiento de la luna en el cielo (ESOfor.122).

Los demás profesores aportan razones asociadas a aspectos conceptuales, especialmente los de Primaria en formación y en menor proporción los de ESO en ejercicio, y también razones clasificadas como "otros", que se centran en la motivación y son más abundantes entre el profesorado en ejercicio.

- Fomenta la comprensión de los movimientos Tierra y Luna (ejemplo de aspectos conceptuales) (ESO-for.133).

- Es necesario que los niños se familiaricen con el modelo para llegar a comprender los procesos (ejemplo de aspectos conceptuales) (Pri-for.155).

- Promueve interés por parte de los alumnos (ejemplo de otros) (ESO-Ejer. 67).

- Los alumnos tienen más curiosidad por este tipo de actividades (ejemplo de otros) (Pri- Ejer.64).

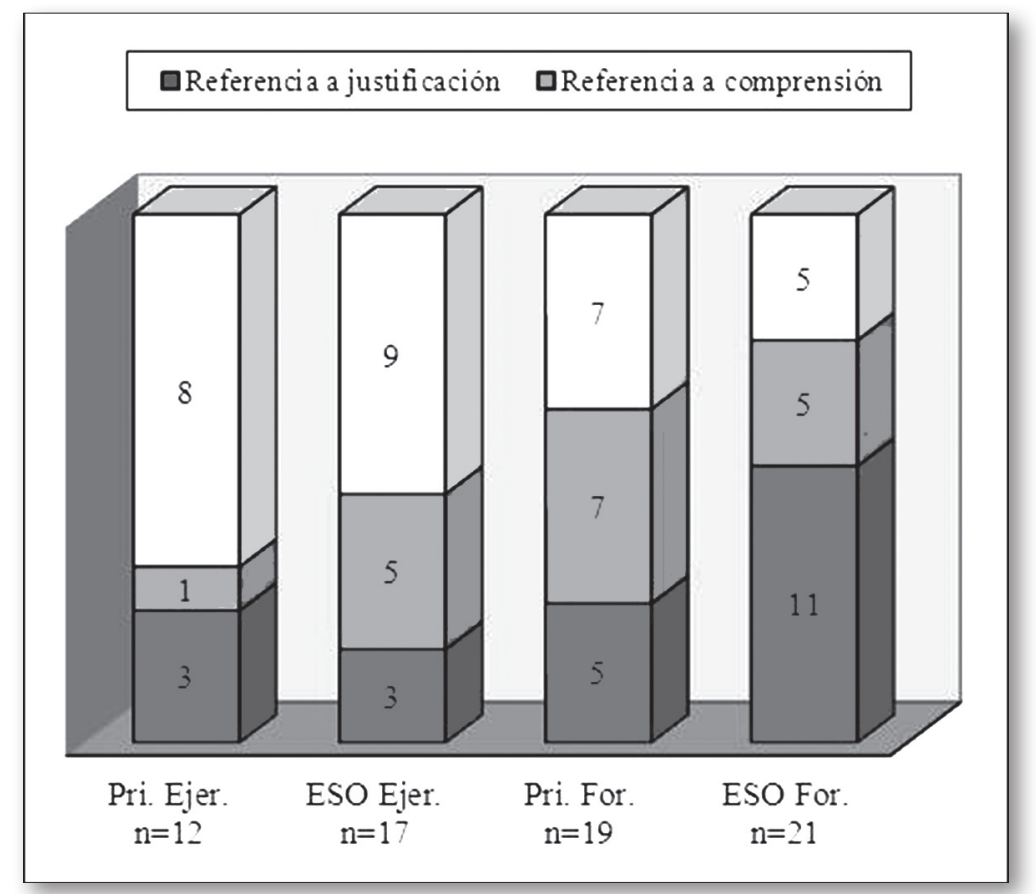

Fig. 3. Justificaciones de los profesores al valorar positivamente la actividad 3.

Las valoraciones negativas no se basan en la habilidad exigida ni se refieren a aspectos conceptuales, por lo que los catalogamos como otros. Los docentes en ejercicio se refieren a la dificultad de la actividad, y los maestros en formación a que no es motivadora:

- Para el alumnado de primaria me parece muy abstracto (Pri-Ejer.103)

- No es una actividad motivadora y no despierta interés en el alumno (Pri-for.148) 


\section{DISCUSIÓN}

Con relación a la primera pregunta que se plantea en este trabajo -¿Cómo valoran los profesores de Primaria y Secundaria en ejercicio y en formación la importancia educativa de distintas habilidades cognitivolingüisticas enunciadas en términos generales y asociadas a la Astronomía? ¿Existen diferencias entre la valoración realizada por los distintos grupos de profesores?-, los resultados obtenidos muestran que las habilidades cognitivo-lingüísticas, al menos de forma declarativa, son ampliamente consideradas por el conjunto del profesorado, siendo las diferencias entre colectivos bastante reducidas o incluso nula. En este sentido, dado que las habilidades encierran distinto grado de dificultad (Jorba, 2000) -describir un proceso observable es, por ejemplo, más fácil que justificar las causas del mismo, empleando un modelo teórico-, cabría esperar mayores diferencias entre las valoraciones realizadas por los grupos de Primaria y Secundaria. Sin embargo, esta no ha sido la tónica general, pues entre los futuros docentes no se hallaron diferencias, y entre los profesores en ejercicio fueron muy limitadas. Estas se asociaron a la descripción de fenómenos, más consideradas por los docentes de Primaria quizás por su sencillez, aunque no se extendieron a una habilidad compleja como la justificación, de la que se esperaría una menor consideración por este último colectivo. Por otra parte, también sería esperable que al comparar los grupos de docentes en ejercicio y en formación del mismo nivel educativo hubiera pocas diferencias. Lo indicado se detecta más en el grupo de Secundaria que en el de Primaria, aunque en ambos se aprecia que los futuros docentes tienden a valorar en mayor medida las habilidades que implican más dificultad -la justificación- que los profesionales, quizás debido a su falta de experiencia directa en relación con las dificultades de los/las niños/as y adolescentes.

Estas escasas diferencias en la valoración de las distintas habilidades cognitivo-lingüísticas por parte de colectivos de niveles educativos diferentes nos conducen a considerar que en la formación docente, y muy especialmente en la inicial, se debería hacer mayor énfasis en las dificultades que encierra cada una de ellas, así como en sus interrelaciones. Si bien es cierto que en Primaria se pueden realizar justificaciones empleando modelos científicos escolares sencillos, tanto en relación con la astronomía como con otros temas (ver currículum oficial), no es menos cierto que encierra dificultades y que se vería favorecida por el dominio de otras habilidades cognitivo-lingüísticas con las que la propia justificación se relaciona (descripción de la realidad, definición de entidades...).

Respecto a la segunda pregunta de este estudio -iLa valoración que realiza el profesorado de las distintas habilidades está influenciada por el contenido?-, el análisis de resultados nos muestra que efectivamente existe tal influencia, pues los docentes priorizan habilidades diferentes en función de que su enunciado sea genérico o esté asociado a un contenido astronómico (cambios diarios/anuales). Así se hallaron diferencias significativas entre la valoración otorgada a una misma habilidad expresada en los términos citados, y también se detectó que los tipos de respuesta más frecuentes, que ordenan las cuatro habilidades en función de su puntuación con independencia de cuál sea ésta, eran diferentes según su correspondencia -enunciados genéricos o astronómicos-. Estas diferencias nos sugieren que el profesorado focaliza su atención, no solo en la habilidad cognitivo-lingüísticas que implica el enunciado de la encuesta, sino también en el contenido científico, informándonos de forma indirecta de cuáles son los conocimientos astronómicos a los que los docentes dan mayor relevancia.

Profundizando en lo indicado, se observa que, en términos generales, y salvo en el grupo de Secundaria en formación, donde apenas se establecen diferencias, en los otros colectivos la descripción de fenómenos se valora sobre todo en enunciados genéricos, mientras que con relación al conocimiento astronómico suele ser menos considerada que las otras. Esta menor importancia otorgada a la descripción de los fenómenos se aprecia muy claramente en el grupo de Primaria en formación, respecto a los enunciados relativos a los cambios anuales. Este resultado es especialmente preocupante, pues este colectivo, en su período formativo, tuvo la oportunidad de discutir la necesidad de insistir, durante el 
proceso de enseñanza, en la descripción de los cambios anuales perceptibles asociados a los cambios de estaciones, recomendándose incluso retrasar a Secundaria la explicación teórica de los mismos, dada su dificultad. Esta requiere destrezas geométricas, junto al dominio de una buena relación entre la perspectiva local y la espacial, difícil incluso para el alumnado de mayor edad (Lanciano y Camino, 2008 ; Gil Quilez y Martínez Peña, 2005). Posiblemente, en la valoración del maestro en formación haya influido más la realización de una actividad dirigida justificar los cambios astronómicos anuales, mediante la simulación del movimiento de traslación con un modelo de esfera/luz (AA.VV., 2009; C. Martínez Losada y García Barros, 2010), que la discusión didáctica asociada a la misma antes mencionada. El uso de la citada simulación suele favorecer la superación de las ideas alternativas, lo que quizás disminuya la percepción de la dificultad que entraña la realización de una adecuada justificación. Esto, unido a la escasa/nula experiencia educativa directa de los futuros maestros, pues no habían asistido al prácticum, explicaría la sobrevaloración de habilidades cognitivo-lingüísticas, más difíciles -descripción del modelo y justificación-, frente a la descripción de cambios observables.

Lo indicado hasta ahora constituye un toque de atención para la formación docente, inicial y permanente, que debe justificar la importancia de las habilidades cognitivo-lingüísticas en el desarrollo del aprendizajes, no solo en términos generales sino también aplicándolos a contenidos específicos. Concretamente, en el caso de astronomía, es imprescindible revalorizar la descripción de los fenómenos regulares - diarios, mensuales, anuales-, pues influyen en la adquisición de conocimientos necesarios para justificar por qué ocurren, vinculando así fenómeno y modelo teórico y dando a este último su verdadero sentido (Navarro, 2011; Shen y Confrey, 2010). Además la descripción del fenómeno observable tiene un valor en sí misma, especialmente para los niveles educativos obligatorios, en la medida que ayuda a adquirir un conocimiento práctico, asociado al movimiento aparente de los astros en el cielo, a la identificación de los puntos cardinales, a la orientación en el espacio y en el plano, etc. Esta insistencia en la astronomía descriptiva, reconocida en el currículum oficial de Primaria y Secundaria, ha sido reivindicada y justificada por diversos autores (Shen y Confrey, 2010; Navarro, 2011; Martínez Losada y García Barros, 2010). Sin embargo nuestra experiencia coincide con Shen y Confrey (2010), en el sentido de que el profesorado se siente incómodo enseñando el citado movimiento aparente de los astros, porque inducen a una interpretación geocéntrica errónea que luego es necesario "desmontar".

Finalmente, con relación a la tercera cuestión planteada en este trabajo - ¿Cómo valoran los docentes las habilidades cognitivo-lingüisticas concretas que demandan determinadas actividades dirigidas al estudio de la Astronomía?-, cabe señalar que el profesorado, especialmente en ejercicio, suele realizar una valoración genérica, al igual que se detectó en otros trabajos (García Barros y Martínez Losada, 2011). Sin embargo, las respuestas más concretas muestran que los participantes no siempre se refieren a la habilidad cognitivo-lingüística específica que la actividad demanda, sino que se centran en el conocimiento conceptual/teórico o en la habilidad cognitiva que se pone en juego en el desarrollo de esta. Así, en la primera actividad sometida a la consideración de los participantes, varios de ellos valoran el conocimiento del concepto satélite, en vez de su definición; en la segunda, la observación y el conocimiento de cómo cambia la posición y forma de la Luna con el paso de las horas, en vez de su descripción, y en la tercera actividad se tiende a destacar sobre todo la comprensión del fenómeno. Esto nos sugiere que el docente, al igual que ocurría en las cuestiones cerradas, no focaliza su valoración en las habilidades cognitivo-lingüísticas o quizás ni siquiera las identifique, problema éste que ha detectado en otro trabajo (Martínez Losada, García Barros y Jiménez-Liso). Por ello es importante que la formación docente haga mayor hincapié en la identificación de las habilidades comunicativas empleando actividades concretas (Ospina Quintero y Bonan, 2011), con el fin de que el docente les otorgue su verdadero valor educativo y reconozca la necesidad de promoverlas explícitamente, pues las personas sabemos "algo" cuando somos capaces de expresarlo correctamente (Sanmartí, 2002). 


\section{CONCLUSIONES}

a) Los distintos colectivos de profesores, participantes en este estudio, valoran positivamente las habilidades cognitivo-lingüísticas presentadas tanto en términos genéricos como astronómicos. Además no se detectan grandes diferencias entre estos.

b) La valoración de las habilidades cognitivo-lingüísticas realizada por los grupos de profesores suele estar influida por el contenido científico al que se asocia su enunciado.

c) Los profesores, aunque consideran importantes las habilidades cognitivo-lingüísticas, tienen dificultades para referirse explícitamente a ellas cuando valoran actividades en las que se exige dichas habilidades.

Estas conclusiones son una aportación más al conocimiento del pensamiento docente, que sin duda debe completarse con otros estudios que permitan profundizar en él mediante, por ejemplo, el análisis de las actividades que seleccionan y las cuestiones de evaluación que proponen, tanto los docentes en su ejercicio profesional como los futuros profesores en el proceso formativo; la observación directa del desarrollo de clases, etc. Sin embargo pueden servir como un primer toque de atención a la formación docente, en la que seguimos profundizando. En ella, como se ha ido apuntando en el apartado anterior, se debe insistir en la necesidad de enseñar en el aula de Primaria y Secundaria las distintas habilidades cognitivo-lingüísticas de forma equilibrada a través de actividades dirigidas al estudio de diferentes tópicos, pues todas ellas contribuyen a la adquisición de competencias científica y también lingüística. La determinación de la progresión de aprendizajes dentro de los grandes temas en los que se organizan los contenidos curriculares de ciencias en la educación obligatoria, puede ser un importante punto de referencia para establecer la correspondiente y paralela progresión en el uso de habilidades cognitivo-lingüísticas, que lógicamente debe ir siendo paulatinamente más exigente.

Este trabajo ha sido financiado por la Xunta de Galicia (Proxecto-código INCITE08XIB106098PR)

\section{BIBLIOGRAFÍA}

AA.vv. (2009). El aprendizaje del modelo Sol-Tierra. Una oportunidad para la formación de los maestros. Alambique, 61, 27-37.

Benarroch, A. y Marín, N. (2011). Relaciones entre creencias sobre enseñanza, aprendizaje y conocimiento de Ciencias. Enseñanza de las Ciencias, 29(2), 289-230.

Cañas, A., Martín-Díaz, M. y Nieda, J. (2007). Competencia en el conocimiento y la interacción con el mundo físico. La Competencia científica. Madrid: Alianza.

De Pro, A. (1998). ¿Se pueden enseñar contenidos procedimentales en las clases de ciencias? Enseñanza de las Ciencias, 16(1), 21-41.

Fuentes, M.J., García Barros, S. y Martínez Losada, C. (2009). ¿En qué medida cambian las ideas de los futuros docentes de secundaria sobre qué y cómo enseñar después de un proceso formativo? Revista de Educación, 349, 269-294.

García Barros, S. y Martínez Losada, C. (2011). ¿Cómo valoran los profesores en ejercicio y en formación unas actividades dirigidas al estudio de la reproducción en la educación obligatoria? . Enseñanza de las Ciencias, 29(1), 5-21.

García Barros, S., Martínez Losada, C., Mondelo, M. y Vega, P. (1997). La Astronomía en los textos escolares de Educación Primaria. Enseñanza de las Ciencias, 15(2), 225-232. 
Gil Quilez, M.J. y Martínez Peña, B. (2005). El modelo de Sol-Tierra-Luna en el lenguaje inográfico de estudiantes de magisterio. Enseñanza de las Ciencias, 23(2), 153-166.

Gómez, I. (2000). Bases teóricas de una propuesta didáctica para favorecer la comunicación en el aula. En J. Jorba, I. Gómez \& A. Prat (Eds.), Hablar y escribir para aprender. Uso de la lengua en situación de enseñanza-aprendizaje desde las áreas curriculares: ICE Universitat Autònoma de Barcelona. Síntesis.

Guisasola, J. y Morentin, M. (2010). Concepciones del profesorado sobre visitas escolares a museos de ciencias. Enseñanza de las Ciencias, 28(1), 127-140 .

Harlen, W. y Qualter, A. (2009). The teaching of science in primary schools. Abingdon: Routledge.

Izquierdo, M. y Adúriz Bravo, A. (2003). Epistemological Foundations of School Science. Science \& Education, 12(1), 27-43.

Izquierdo, M. y Aliberas, J. (2004). Pensar, actuar i peular a la clase de ciències. Por un ensenyament de les ciències racional i raonable.: Universitat Autónoma de Barcelona.

IzQuierdo, M. y SANMARTí, N. (2000). Enseñar a leer y escribir textos de Ciencias de la Naturaleza. En J. Jorba, I. Gómez y A. Prats (eds.), Hablar y escribir para aprender. Uso de la lengua en situación de enseñanza-aprendizaje desde las áreas curriculares (pp. 181-200). Barcelona: ICE Universitat Autònoma de Barcelona. Síntesis.

Izquierdo, M., Sanmartí, N. y Espinet, M. (1999). Fundamentación y diseño de las prácticas escolares de ciencias experimentales. Enseñanza de las Ciencias, 17(1), 45-59.

http://dx.doi.org/10.1023/A:1022698205904

Jorba, J. (2000). La comunicación y las habilidades cognitivo-lingüísticas . In J. Jorba, I. Gómez y A. Prat (eds.), Hablar y escribir para aprender. Uso de la lengua en situación de enseñanza-aprendizaje desde las áreas curriculares (pp. 29-49). Barcelona: ICE Universitat Autònoma de Barcelona. Síntesis.

Lanciano, N. y Camino, N. (2008). Del ángulo de la geometría a los ángulos en el cielo. Dificultades para la conceptalización de las coordenadas astronomicas acimut y altura. . Enseñanza de las Ciencias, 26(1), 77-92.

Martínez Losada, C. y García Barros, S. (2005). Do Spanish secondary school teachers really value different sorts of procedural skills? International Journal of Science education, 7 (3), 827-854. http://dx.doi.org/10.1080/09500690500038355

Martínez Losada, C. y García Barros, S. (2010). Qué vemos en el cielo y cómo podemos explicarlo. En A. De Pro (ed.), Competencias en el conocimiento e interacción con el mundo físico: La comprensión del entorno próximo (pp. 163-196). Madrid: MEC.

Martínez Losada, C., García Barros, S. y Jiménez-Liso, R. (2011, 5-9 septiembre). The Cognitive and Linguistic Skills Associated to Human Nutrition in Teacher Training. Paper presented at the ESERA Conference, Lyon.

Martínez PeÑA, B. y Gil, M. J. (2001). The importance of images in astronomy education. International Journal of Science Education, 23(11), 1125-1135.

http://dx.doi.org/10.1080/09500690110038611

Navarro, M. (2009). Aprendizaje y enseñanza de astronomía diurna en Primaria Universidad de Alicante.

Navarro, M. (2011). Enseñanza y aprendizaje de astronomía diurna en primaria mediante "secuencias problematizadas" pasadas en "mapas evolutivos". Enseñanza de las Ciencias, 29(2), 163-174.

Ospina Quintero, N., \& Bonan, L. (2011). Explicaciones y argumentos de profesores de quimica en formación inicial: la construcción de criterios para su evaluación. Revista Eureka sobre Enseñanza y Divulgación de las Ciencias 8 (1),(1), 2-19. 
Pérez Esteve, P., \& Zayas, F. (2007). Competencia en comunicación lingüística. Madrid: Alianza Editorial.

Porlán, R., Martín del Pozo, R., Rivero, A., Harres, J., Azcárate, P. y Pizzato, M. (2011). El cambio del profesorado de ciencias II: itinerarios de progresión y obstáculos en estudiantes de magisterio. Enseñanza de las Ciencias, 29(3), 253-270.

Pozo, J. I., Scheuer, N., Mateos, M. y Pérez, M.P. (2006). Las teorías implícitas sobre el aprendizaje y la enseñanza. En J. I. Pozo, N. Scheuer, M. P. Pérez, M. Mateos, E.

Martín y M. De La Cruz (eds.). Nuevas formas de pensar la enseñanza y el aprendizaje, (pp. 95-132. ). Barcelona: Graó.

Prain, V. (2006). Learning from Writing in Secondary Science: Some theoretical and practical implications. International Journal of Science Education, 28(2-3), 179-201. http://dx.doi.org/10.1080/09500690500336643

SAnMartí, N. (2002). Didáctica de las ciencias en la educación secundaria obligatoria. Madrid: Síntesis Educación.

SANMARTí, N. (2007). Hablar, leer y escribir para aprender ciencia. En P. Fernández (ed.). La competencia en comunicación ligüistica en las áreas del currículo. Madrid: Colección Aulas de Verano. MEC.

Sanmartí, N., IzQuierdo, M. y García Rovira, M. P. (1999). Hablar y escribir. Una condición para aprender ciencias. Cuadernos de Pedagogía, 281, 54-58.

Shen, J. y Confrey, J. (2010). Justifying Alternative Models in Learning Astronomy: A study of K-8 science teachers' understanding of frames of reference. International Journal of Science Education, 32(1), 1-29.

http://dx.doi.org/10.1080/09500690802412449

Solbes, J. y Palomar, R. (2011 ). ¿Por qué resulta tan difícil la comprensión de la astronomía a los estudiantes?. Didáctica de las Ciencias Experimentales y Sociales, 25, 187-211.

Sutton, C. (1996). Beliefs about science and beliefs about language. International Journal of Science Education, 18, 1-18.

http://dx.doi.org/10.1080/0950069960180101

Sutton, C. (2003). Los profesores de ciencias como profesores de lenguaje. Enseñanza de las Ciencias, 21(1), 21-25.

Trumper, R. (2006). Factors affecting students' junior high school students' interest in physics. Journal of Science Education and Technology, 52-60.

VÁzquez, A., Pelizza, L., Jаков, I. y Rosales, P. (2007). Concepciones de los profesores universitarios acerca de las relaciones entre tareas de escritura y aprendizaje. Disponible en: http://www.filo.unt. edu.ar/jorn_unesco/cd/PAN\%202.3.VAZQUEZ.pdf.

Vosniadou, S. (d1991). Designing curricula for conceptual restructuring; lessons from the study of knowledge acquisition in astronomy. Journal of Curriculum Studies, 23, 219-237. 


\section{ANEXO I.}

\section{Encuesta}

1. De las siguientes habilidades relacionados con el aprendizaje y su comunicación, valora de 1 a 5 (1 ninguna,.... 5 mucha) Su importancia educativa.

- Describir hechos y fenómenos naturales, observables (movimiento de objetos, arco iris, ..). (GDF)

- Describir procesos no observables (digestión, conservación de la masa en los cambios químicos...) o de modelos científicos (modelo de célula, de Tierra...) (G-DM).

- Definir conceptos (célula, disolución, animal, ..) (G-Def).

- Justificar hechos y fenómenos empleando un modelo científico (justificar el cambio de estado desde un modelo de partículas en movimiento, ...) (G-J).

2.- A continuación se enuncian una serie de conocimientos relativos a aspectos astronómicos que implican una comunicación: descripciones de hechos/fenómenos o de modelos científicos; definiciones de conceptos y explicaciones/justificaciones de hechos/fenómenos. Valora de 1 a 5 (1 ninguna/ nunca, .... 5 mucha/siempre) la importancia educativa de las habilidades concretas implicadas en cada enunciado.

- El Sol aparentemente se mueve en el cielo de este a oeste, es decir, cambia de posición respecto a un punto de referencia (un árbol, poste...). (D-DF)

- En nuestra zona geográfica, el número de horas de luz y la altura del Sol al mediodía varía a lo largo del año. El 21 de diciembre el día es más corto y el Sol está más alto, y el 21 de junio es el día es más largo y el Sol está mas bajo. (A-DF)

- La Tierra tiene forma esférica, gira sobre si misma (D-DM).

- La Tierra gira alrededor del Sol y el eje de rotación terrestre está inclinado respecto al plano de su órbita de traslación (A-DM).

- La rotación terrestre es el movimiento de la Tierra alrededor de su eje (D-Def).

- La traslación terrestre es el movimiento de la Tierra alrededor del Sol (A-Def).

- El Sol se mueve aparentemente en el cielo a lo largo del día, porque la Tierra gira sobre si misma (movimiento de rotación). Esto permite que la superficie terrestre se vaya iluminando y oscureciendo a lo largo del día (D-J).

- En nuestra zona geográfica el número de horas de luz/oscuridad y la altura del Sol al mediodía cambian regularmente a lo largo del año, debido a la inclinación del eje de la Tierra y a su movimiento de traslación alrededor del Sol. Así en un determinado momento nuestro hemisferio está orientado hacia el Sol, los rayos caen más perpendicularmente, es verano. Cuando la Tierra se halla en la parte opuesta de la órbita, nuestro hemisferio no está orientado hacia el sol, los rayos caen más oblicuos, es invierno (A-J).

Nota. Se identifica cada enunciado con las siglas empleadas en el trabajo, que no se incluyeron en la encuesta

3.- Las tres actividades que se recogen a continuación, están dirigidas al estudio de la Luna. Cada una de ellas permite trabajar diferentes habilidades relacionadas con la comunicación. Comenta lo mas detalladamente posible el interés educativo de las mismas. 


\section{Actividad 1}

Se dice que la Tierra es un planeta y la Luna es un satélite. ¿Podrías dar una definición de planeta? ¿y de satélite?

\section{Actividad 2}

Hoy podemos ver la Luna al llegar a clase: ¿cuál es su forma?; ¡a qué letra se parece?; ¡en qué punto cardinal está?

¿La Luna estará en la misma posición dentro de una hora? y mañana a esta misma hora ¿estará en el mismo sitio? ¿Qué forma tendrá?

Para contestar a estas preguntas debes hacer las correspondientes observaciones. Es importante que tomes un punto de referencia (un poste, árbol...). Describe los cambios que has observado: hacia qué punto cardinal se movió, como es su forma.

\section{Actividad 3}

La Luna, al igual que el Sol, cambia de posición en el cielo a lo largo del día. Nace por el este y se pone por el oeste. Además la salida de la luna se retrasa diariamente unos 45 minutos (los periódicos informan ello).

Basándote en los movimientos que realmente realizan la Tierra y la Luna, explica:

- el cambio de posición de la Luna desde que sale hasta que se pone.

- que la salida de la Luna se retrase diariamente 45 minutos.

Para eso podes utilizar un modelo de esferas que representen los dos astros y sus movimientos. 


\title{
The importance of the cognitive-linguistic skills related to the study of the Astronomy from the teacher's view
}

\author{
Susana García Barros, Cristina Martínez Losada \\ Departamento de Pedagoxía e Didáctica. \\ Universidade da Coruña \\ susg@udc.es, cmarl@udc.es
}

This essay aims to answer the following questions:

- How much do Primary and Secondary school teachers (those already working and those in training) value the educational importance of various cognitive-linguistic skills, when related to general terms and to terms relating to Astronomy? Are there any differences between the values given by the different groups of teachers?

- Are the teachers' evaluations of various skills influenced by content?

- How much do the teachers value the specific cognitive-linguistic skills required by certain activities aimed at the study of Astronomy?

The value of communication in the study of sciences and the need for the student to produce different texts is justified in the theoretical framework. It also shows how the various cognitive-linguistic skills are used, skills such as the description of facts/phenomena and theoretical models, the definition of concepts, and the justification of the cited facts/phenomena. It is worth noting that the acquisition of cognitive-linguistic skills is related to the scientific content. In particular, this study uses astronomy as it is a subject which is included in both Primary and Secondary education. The subject alludes to both observational and descriptive aspects of what we see in the sky, as well as the explicative aspect which relates the observed phenomena to theoretical models.

155 teachers participated in the study: 59 professional with experience in the 2nd and 3rd cycle of Primary; 54 teachers from the first cycle of the Secondary (ESO) exam; 21 Primary teachers in training and 21 future teachers of Secondary education. The gathering of data was done using a survey which had two parts. In the first part, the participants had to give a value of 1 to 5 for the educational importance of a series of cognitive-linguistic skills: the description of phenomena or facts; the description of theoretical models; and the definition and justification of facts/ phenomena. These skills were compared using generic statements accompanied by examples and astronomical statements regarding the study of daily changes and their explanation, and also annual changes and their explanation. In the second part, the participants had to answer open questions, evaluating the cognitive-linguistic skills required in three activities based on the study of the Moon: 1) the definition of a satellite; 2) description - visible changes in position over 24 hours; 3) the justification of the changes, using the Sun/Earth/Moon model.

The answers to the closed questions were analysed statistically. To detect the differences between the groups of teachers with regards to the value awarded to each statement, the $\chi^{2}$ Pearson test was used. To find out the differences between the two types of statements, as established by each collective, the McNemar test was used (Value of significance in both cases, $\mathrm{p}<0.05$ ).

The values of the skills required for the activities, with regards to the open questions, were classified based on what the value referred to: a) explicitly to the cognitive-linguistic skill required by the activity and b) to other aspects such as concepts dealt with in the activity or to other skills involved in it.

The results show that the groups of teachers positively value the cognitive-linguistic skills shown in both the generic statements and in the astronomical ones. Also, large differences were not detected between groups, despite the fact that they work at different educational levels.

The evaluation of the cognitive-linguistic skills carried out by the groups of teachers is usually influenced by the scientific content associated with the statement. Specifically, the description of phenomena is valued more in generic statements. The description of models and definitions are more highly considered in astronomical terms than in generic ones.

Despite considering cognitive-linguistic skills to be important, the teachers have difficulties in referring to them explicitly when they evaluate the activities in which such skills are required. They generally refer to the conceptual/ theoretical knowledge or the cognitive skill associated with its development.

These results should be born in mind during teacher training. This training should consider the need to teach in Primary and Secondary schools the various cognitive-linguistic skills in a balanced way via activities. It is also important to determine the progression of learning within the relevant subjects of compulsory education, like astronomy, for instance. This progression could be an important reference point to establish parallel progression in the use of cognitive-linguistic skills, which must logically become increasingly demanding. 